\title{
STATUS AND DEVELOPMENT OF THE KAZAKHSTAN ISOCHRONOUS CYCLOTRON
}

\author{
A. Arzumanov, V. Batischev, A. Borissenko, M. Gorkovets, V. Koptev, S. Lyssukhin, S. Mulgin, \\ Yu. Popov, S. Zhdanov, Institute of Nuclear Physics, Almaty, 480082, Kazakhstan.
}

\section{Abstract}

A report is presented on the status of the cyclotron in Almaty at present time. In spite of sharp curtailment of government-funded basic research programme the cyclotron still remains as multipurpose accelerator facility and is being used for fundamental research and application in different fields of applied science.

During last years some cyclotron systems were upgraded and some were designed anew. The system for on-line precise ion beam energy measurement using timeof-flight method was designed and constructed. Inductive pick-up electrodes are integrated into beam transport channel and are located at the distance of $10 \mathrm{~m}$ from each other. Average beam energy determination is performed with the accuracy about $0.1 \%$. This system allows to analyse ion beam parameters in pulse-mode of cyclotron operation with macro duty factor in the range of $2-50$.

In connection with development of cyclotron based radioisotope production and necessity of irradiation of toxic materials and loose and smelted powders with low heat conductivity circular beam scanning system was designed and installed. Transversal rotating magnetic field is generated by stator of usual electro-motor. The scanner allows to form predictable beam power density distribution on the target surface and obtain rather uniform irradiation fields.

Cyclotron based neutron source with Be target for research on radioecology and neutron dosimetry was constructed and is located at one of the external beam transportation channel. Parameters of the system and examples of its application are presented.

\section{INTRODUCTION}

The isochronous cyclotron in Almaty is a compact low energy cyclotron [1]. It generates different beams of light ions: protons $6-30 \mathrm{MeV}$, deuterons $12.5-25 \mathrm{MeV},{ }^{3} \mathrm{He}$ ions $18.5-62 \mathrm{MeV}$, alpha particles $25-50 \mathrm{MeV}$. The cyclotron is operated in pulse mode at duty factor of $2-$ 50. The main beam parameter - energy of accelerated particles, is monitored with upgraded system based on using of pick-up electrodes and time-of-flight technique [2]. Modern apparatus provides precise measurements of electric signals induced by accelerated particle bunches and allows to determine average beam energy with $0.1 \%$ accuracy.
In the last years the cyclotron is rather intensively used for radioisotopes production. For irradiation of targets containing toxic materials in the form of powders the circular beam scanning system was developed and installed in the beam transport channel. Rotating transversal magnetic field with revolution rate of $50 \mathrm{~Hz}$ allows to shift the beam spot on the target for up to 12 $\mathrm{mm}$ and to form rather homogeneous beam power distribution on the irradiated surface.

The cyclotron based thermal neutron source was developed and mounted in the cyclotron beam line for radioecological application. The analytical method of thermal neutron induced fission, developed with help of this facility, allows to study spatial distribution of ${ }^{235} \mathrm{U}$ and ${ }^{239} \mathrm{Pu}$ impurities in the non-fissile samples from test sites in Kazakhstan.

\section{CYCLOTRON BEAM ENERGY MEASUREMENT BY TIME-OF-FLIGHT METHOD}

The time-of-flight technique of beam energy measurement for pulse mode of cyclotron operation was developed. The cylindrical pick-up electrodes with $98 \mathrm{~mm}$ internal diameter and $100 \mathrm{~mm}$ length were integrated into the cyclotron beam line at the distance of $9352 \mathrm{~mm}$. The electrodes are designed as non-symmetrical striplines enveloping ion beam with impedance of $50 \Omega$. Low impedance allows to decrease noise RF-voltages from the acceleration system and to sharp signals of beam bunches (Fig 1). Pulses from pick-up electrodes are transmitted via RF-cables of equal electrical length to the measuring unit which consists of amplifiers with amplification factor of $25 \mathrm{~dB}$, filters and time intervals measuring device.

Electronic tests and experiments with ion beam demonstrated following performance parameters of the energy measurement system:

- Sensitivity $-0.7 \mathrm{mV} / \mu \mathrm{A}$;

- Uncertainty of time intervals measurement - \pm 50 ps;

- $\quad$ Frequency bend $-0.05-500 \mathrm{MHz}$.

Ion beam energy calculation is performed using the equation:

where:

$$
\begin{gathered}
E=E_{0}\left[1 /\left(1-\beta^{2}\right)^{1 / 2}-1\right] ; \\
\beta=v / c ; \\
v=L /[T(n-1)+\Delta t] ;
\end{gathered}
$$

$L-$ the distance between pick-up electrodes; 
$T$ - period of bunches sequence;

$n$ - number of bunches along measuring base;

$\Delta t$ - the part of total time of bunch flight between pickup electrodes which is measured with apparatus.

Total error in determination of average beam energy depends on accuracy of measurement of flight base length, measurement of time intervals and asymmetry of signal cables. Uncertainty of average energy measurement in this case does not exceed $0.1 \%$.

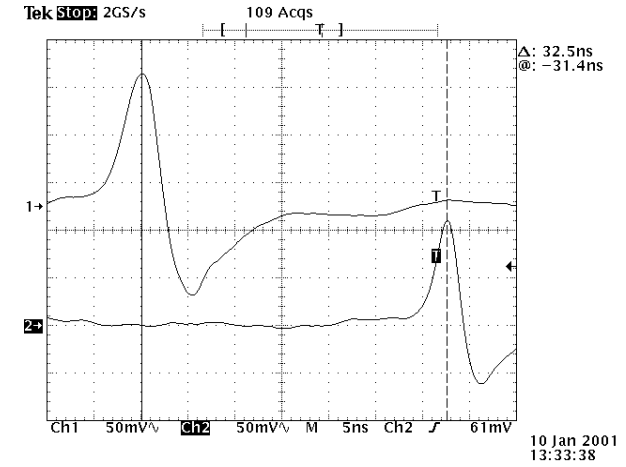

Figure 1: Signals of pick-up electrodes for $29 \mathrm{MeV}$ alphaparticles bunches.

\section{CIRCULAR BEAM SCANNING SYSTEM FOR RADIOISOTOPES PRODUCTION}

During some last years an essential work was performed on upgrading and modernization of the cyclotron in order to provide reliable radioisotopes production. At the time being the accelerator is equipped with two facilities for isotope target irradiation using internal and external cyclotron beams.

The internal isotope target system is intended for irradiation works with high power proton beams up to 10 $\mathrm{kW}$ [3], so irradiated surface must have very good heat conductivity. Normally it is thin metal layers on the copper backing.

The external isotope target system is used for irradiation of toxic materials and materials with low heat conductivity such as loose and smelted powders [4]. In this case even using low power ion beam $(<100 \mathrm{~W})$ may lead to local overheating of the target surface due to inhomogeneous beam current density distribution and long term instability of this distribution (Fig 2).

The circular electromagnetic scanning system was designed and constructed for formation of the predictable beam power distribution. Transversal rotating magnetic field with revolution rate of $50 \mathrm{~Hz}$ is generated by the stator of usual three-phase industrial electro-motor and provides beam declining from the transport channel axis. The stator is mounted on the external cyclotron beam line and placed at the distance of about 3 meters from the isotope target. Maximum value of magnetic field at the stator axis is $300 \mathrm{G}$ with non-uniformity along diameter less than $3 \%$. The scanning system allows to obtain 12 $\mathrm{mm}$ beam spot offset from the center of the target surface for $30 \mathrm{MeV}$ protons. Thus, it is easily to adjust beam spot size and off-set value so, that to get non-uniformity of power distribution better than $20 \%$ inside of circle with diameter $20 \mathrm{~mm}$ which corresponds to isotope target dimension (Fig. 3). Edges of ion beam are removed by graphite diaphragm to protect the target holder from overheating.

Control of beam profile and size of scanning is carried out by using TV-system and movable probe equipped with Be-oxide disk, located near the target.
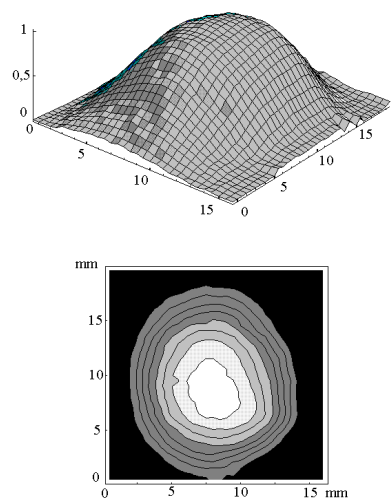

Figure 2: The current density distribution of focussed ion

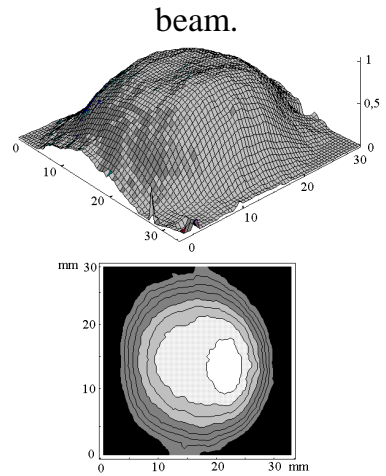

Figure 3: Scanned beam power distribution at the target surface.

\section{CYCLOTRON SOURCE OF THERMAL NEUTRONS FOR RADIO-ECOLOGICAL APPLICATION}

At the time being and in the longterm perspective the radio-ecological situation at the nuclear test sites in Kazakhstan will be mainly dependent on radioactivity of some products of fission reactions (mainly radioisotopes ${ }^{90} \mathrm{Sr},{ }^{137} \mathrm{Cs}$ ) as well as spread material of nuclear charge of the bombs - radionuclides ${ }^{239} \mathrm{Pu},{ }^{235} \mathrm{U}$. From mentioned nuclides the highest danger for human health presents alpha-radioactive isotope ${ }^{239} \mathrm{Pu}$.

One of the most sensitive methods for determination of trace concentration and spatial distribution of ${ }^{239} \mathrm{Pu}$ and ${ }^{235} \mathrm{U}$ is based on thermal neutron induced fission and registration of fission products by the fragment detectors. Minimal sample preparation need for this kind of measurement gives the unique opportunity for study of 
trace radioisotope impurities distribution at the ground surface and registration of "hot" radioactive particulates.

For realisation of this analytical method the generator of thermal neutrons was developed, constructed and has been built into the cyclotron beam line. The generator consists of the two main parts: beam target - source of fast neutrons from nuclear reactions, and block-moderator of neutrons (Fig. 4).

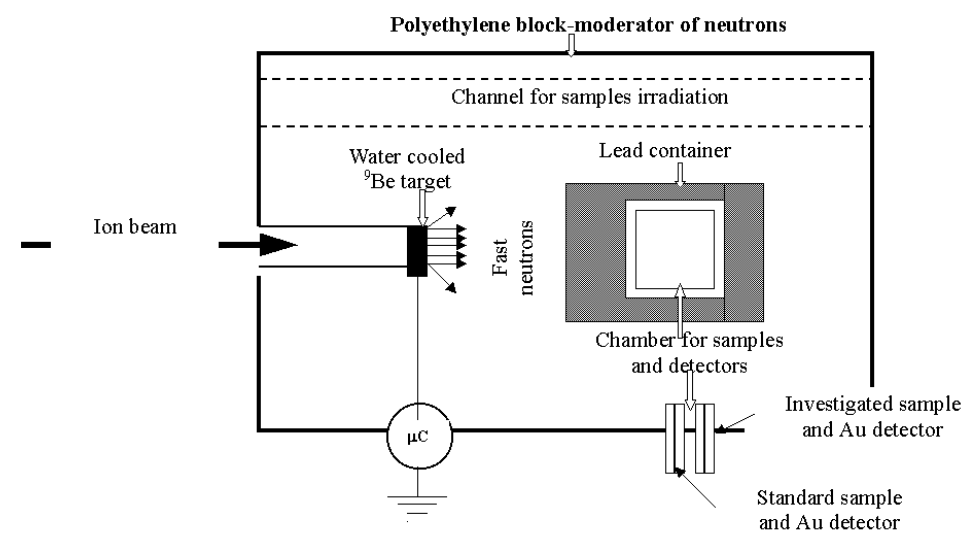

Figure 4: Block-scheme of the thermal neutron source.

The target device is made of Be-plate by $10 \mathrm{~mm}$ thickness integrated into the water cooled aluminium target holder. The block-moderator is manufactured of polyethylene cube with $880 \mathrm{~cm}$ side and is equipped with the channels for samples irradiation and the lead container for the experiments with reduced gamma-background.

Irradiation of the samples was done using reaction ${ }^{9} \mathrm{Be}(\mathrm{d}, \mathrm{xn})$ for generation of initial neutron flux with average energy about $1.5 \mathrm{MeV}$. Flux of thermal neutrons was $2 \cdot 10^{10} \mathrm{ncm}^{-2} \mathrm{~s}^{-1}$ at the deuteron beam current $12 \mu \mathrm{A}$ with energy of $25 \mathrm{MeV}$. The thermal neutron flux measurements were carried out by the standard gold detectors.

A typical distribution of fission fragment tracks in the glass detector with magnification factor of 100 is shown in Figure 5.

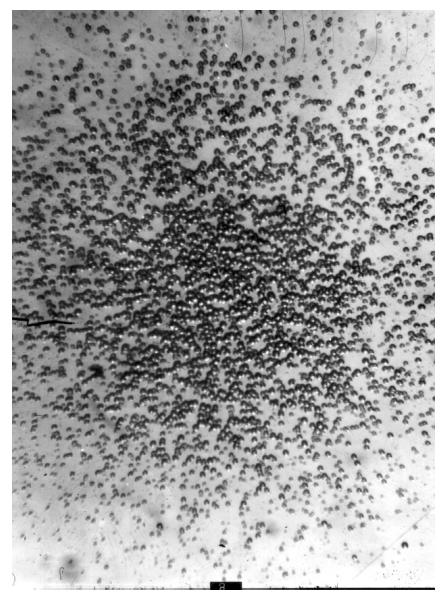

Figure 5: The typical distribution of the fission fragment tracks in the detector at magnification factor of 100 .

\section{CONCLUSION}

In connection with growing interest in the cyclotron based applied research - radioisotope production and radioecology, the isochronous cyclotron in Almaty was upgraded. New circular beam scanning system resulted in rather homogeneous external isotope target irradiation. Time-of-flight measurement system allowed to control extracted ion beam energy with accuracy $0.1 \%$. Developed cyclotron based thermal neutron source gives an opportunity for precise determination of ${ }^{239} \mathrm{Pu}$ and ${ }^{235} \mathrm{U}$ in the samples from nuclear test sites. The cost of such analysis is much low in comparison with nuclear reactor experiments.

Newly developed techniques essentially increased flexibility and efficiency of the cyclotron applications.

\section{ACKNOWLEDGEMENTS}

The authors would like to express their gratitude to ISTC for support of this work.

\section{REFERENCES}

[1] A.A. Arzumanov, L.M. Nemenov, Nucl. Instr. and Meth., 1973, vol. 106, N2, $201-202$.

[2] D.A. Lind, R.J. Peterson etc., Nucl. Instr. and Meth., A292 (1990), 247 - 253.

[3] A.A. Arzumanov, V.N. Batischev etc., "Radioisotope Production at the Cyclotron in Almaty", Proc. XV Int. Conf. Cycl. Appl., Caen, France, June, 1998.

[4] A.A. Arzumanov, V.N. Batischev etc., "Production of plutonium, yttrium and strontium tracers for using in environmental research", this Conf.

[5] S.P. Tretyakova, Phys. Part. Nuclei, 1992, v. 23, N2. 